\title{
Contrasting Plasmodium infection rates and insecticide susceptibility profiles between the sympatric sibling species Anopheles parensis and Anopheles funestus s.s: a potential challenge for malaria vector control in Uganda
}

Charles Mulamba ${ }^{1,2}$, Helen Irving ${ }^{1}$, Jacob M Riveron¹, Louis G Mukwaya², Josephine Birungi ${ }^{2}$ and Charles S Wondji ${ }^{*}$

\begin{abstract}
Background: Although the An. funestus group conceals one of the major malaria vectors in Africa, little is known about the dynamics of members of this group across the continent. Here, we investigated the species composition, infection rate and susceptibility to insecticides of this species group in Uganda.

Methods: Indoor resting blood-fed Anopheles adult female mosquitoes were collected from 3 districts in Uganda. Mosquitoes morphologically belonging to the An. funestus group were identified to species by PCR. The sporozoite infection rates were determined by TaqMan and a nested PCR. Susceptibility to major insecticides was assessed using WHO bioassays. The potential role of four candidate resistance genes was assessed using qRT-PCR.
\end{abstract}

Results: An. funestus s.s. and An. parensis, were the only members of the An. funestus group identified. Both species were sympatric in Masindi (North-West), whereas only An. parensis was present in Mityana (Central) and Ntungamo (South-West). The Plasmodium falciparum infection detected in An. parensis (4.2\%) by TaqMan could not be confirmed by nested PCR, whereas the 5.3\% infection in An. funestus s.s. was confirmed. An. parensis was susceptible to most insecticides, however, a moderate resistance was observed against deltamethrin and DDT. In the sympatric population of Masindi, resistance was observed to pyrethroids (permethrin and deltamethrin) and DDT, but all the resistant mosquitoes belonged to An. funestus s.s. No significant over-expression was observed for the four P450 candidate genes CYP6M7, CYP9K1, CYP6P9 and CYP6AA4 between deltamethrin resistant and control An. parensis. However, when compared with the susceptible FANG An. funestus s.s strain, the CYP9K1 is significantly over-expressed in An. parensis (15-fold change; $\mathrm{P}<0.001$ ), suggesting it could play a role in the deltamethrin resistance.

Conclusion: The contrasting infection rates and insecticide susceptibility profiles of both species highlights the importance of accurate species identification for successful vector control programs.

Keywords: An. parensis, An. funestus, Malaria, Insecticide resistance, Vector control, Uganda

\footnotetext{
* Correspondence: c.s.wondji@liverpool.ac.uk

${ }^{1}$ Vector Biology Department, Liverpool School of Tropical Medicine,

Pembroke Place, Liverpool L3 5QA, UK

Full list of author information is available at the end of the article
} 


\section{Background}

Malaria control in Africa, notably in Uganda, has seen a significant scale up of vector control interventions such as Long Lasting Insecticide Nets (LLINs) and Indoor Residual Spraying (IRS) [1]. The monitoring of the efficacy of these programs requires a good understanding of the vector composition in areas under control. One of the main malaria vectors in Uganda, Anopheles funestus s.s. has been found to be resistant to both pyrethroids and DDT in Eastern Uganda [2], highlighting the need to closely monitor this vector to ensure the continued success of control interventions. However, An. funestus belongs to the Anopheles funestus group which comprises nine to eleven morphologically indistinguishable species at the adult stage; An. funestus s.s, An. parensis, An. confusus, An. funestus-like, An. aruni, An. vaneedeni, An. leesoni, An. brucei, An. rivulorum, An. rivulorum-like, and An. fuscivenosus [3-7]. An. funestus s.s, An. parensis, An. vaneedeni and $A n$. aruni Sobti have identical morphology at all or some life stages and are referred to as the funestus sub-group [4,5]. The other members of the group exhibit distinctive characteristics at different stages $[8,9]$.

An. funestus S.s. and An. rivulorum are distributed throughout sub-Saharan Africa [4,5], An. parensis is common in South Africa, Swaziland and eastern Africa, whereas the other members are more localized $[8,9]$.

An. funestus s.s is the most efficient malaria vector in this group [10] and one of the major vectors in Uganda [11], while the other group members are known to be zoophilic with only An. rivulorum having been implicated as a minor vector in Tanzania [10]. An. vaneedeni has been shown to transmit parasites under laboratory conditions but has not been associated with malaria transmission in nature [12].

Due to the significant differences existing between the members of the An. funestus group in terms of their vectorial capacities, resting and biting behaviour and despite their close morphological similarities [13], it is important to accurately identify mosquitoes of this group and establish their geographical distribution. This is particularly important in order to assess their respective contribution to malaria transmission and their susceptibility to insecticides used by control programs. In Uganda, if An. funestus s.s. has been previously described in the country, little is known about the existence of other members of the $A n$. funestus group. Their presence, geographical distribution, their resting behaviour, their vectorial capacity and their susceptibility to main insecticides used in public health remain poorly characterised. In an attempt to fill these gaps of knowledge, we report here the characterisation of the An. funestus group from 3 locations in Uganda, particularly their species composition, their contribution to malaria transmission and their susceptibility to main public health insecticides.

\section{Methods}

\section{Study sites}

Adult Anopheles mosquitoes were collected from 3 districts in Uganda (Figure 1); Masindi $\left(1.68^{\circ} \mathrm{N}, 31.70^{\circ} \mathrm{E}\right)$, Mityana $\left(0.40^{\circ} \mathrm{N}, 32.03^{\circ} \mathrm{E}\right)$ and Ntungamo $\left(0.88^{\circ} \mathrm{N}, 30.26^{\circ} \mathrm{E}\right)$ as part of an insecticide resistance monitoring program. Villages sampled in these districts are located in close proximity with rivers, swamps and tributaries joining major rivers as these permanent water bodies are suitable breeding sites for $A n$. funestus. The surrounding vegetation is mainly shrubs, maize crops, finger-millet, banana and coffee plantations. Fishing and subsistence farming are the main human activities around these villages. It was of notice that domesticated animals including cattle, goats and sheep were present but on a small scale.

\section{Mosquito collection and rearing}

Indoor resting blood fed adult female Anopheles mosquitoes $\left(\mathrm{F}_{0} \mathrm{~s}\right)$ were collected between $06.00 \mathrm{am}$ and $12.00 \mathrm{pm}$, following verbal consent from the village Local Council 1 (L.C1) chairpersons and household owners. Mosquito collections were carried out between early May and June, 2012. Mosquitoes were collected into netted paper cups using manual aspirators and torches, kept in a cool box and immediately transported to the insectary at the Uganda Virus Research Institute based in Entebbe, Uganda.

A forced-egg laying method described by [2] was used to induce the females to lay eggs. Eggs were stored at room temperature for up to 3 days and were transferred to the Liverpool School of Tropical Medicine (LSTM), UK (under the LSTM import license from DEFRA). The egg batches were allowed to hatch in a small paper cup and later transferred to larvae bowls for rearing as previously described $[2,14]$.

\section{Species Identification}

Field caught females $\left(\mathrm{F}_{0} \mathrm{~s}\right)$ which oviposited first generation $\left(\mathrm{F}_{1}\right)$ egg batches above, were morphologically identified as belonging to An. funestus group according to the key of [4]. The $\mathrm{F}_{0} \mathrm{~s}$ were split into two portions (head + thorax and abdomen). Genomic DNA was extracted from head and thorax (including proboscis) using the Livak method described in [15], while the abdomen was reserved for possible future investigations. A cocktail PCR described by [16] was performed to identify member species of the $A n$. funestus group with the use of an An. funestus specimen as positive control.

\section{Estimation of the sporozoite infection rate}

The sporozoite infection rate was determined using the TaqMan assay described by [17]. The real-time PCR MX 3005 (Agilent, Santa) system was used for amplification. $1 \mu \mathrm{l}$ of gDNA for each sample was used as a template in 


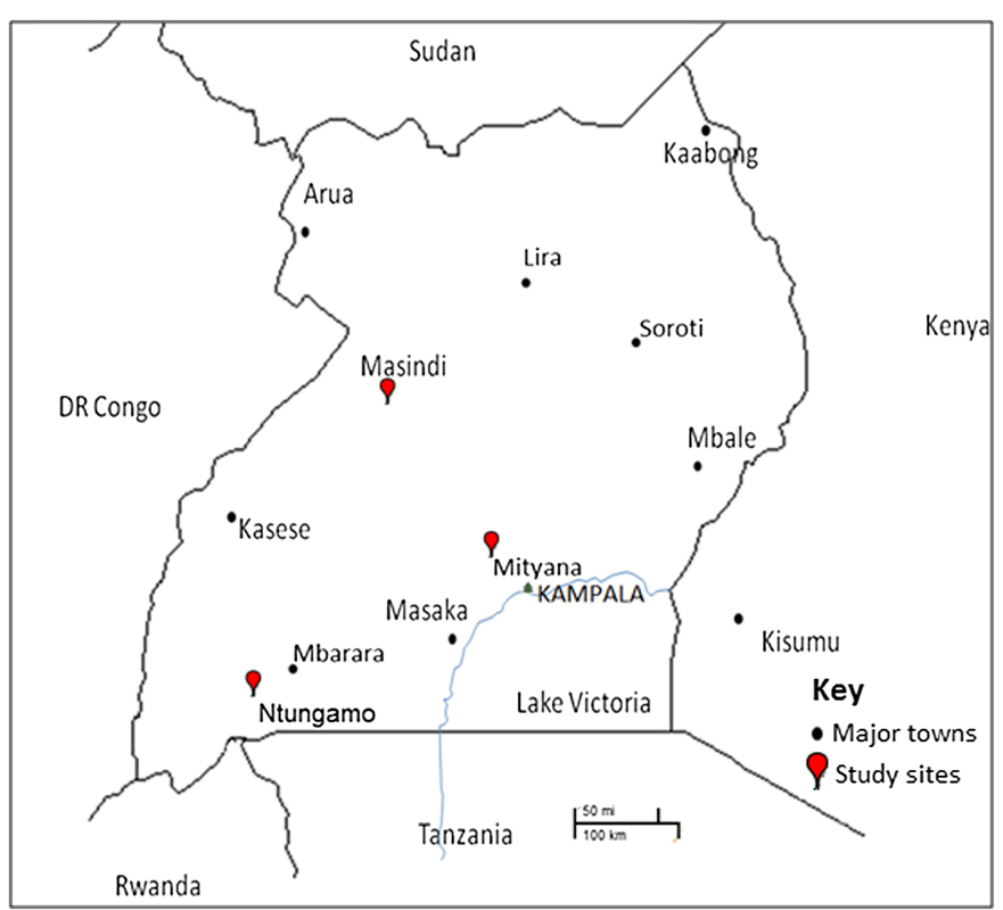

Figure 1 Map of Uganda showing the three study districts (with the red indicator). Major towns in Uganda are also included for further guidance.

a 3-step program, with a denaturation at $95^{\circ} \mathrm{C}$ for 10 mins, followed by 40 cycles of $15 \mathrm{sec}$ at $95^{\circ} \mathrm{C}$ and $1 \mathrm{~min}$ at $60^{\circ} \mathrm{C}$. Primers described by [17] were used together with two probes labelled with fluorophores, FAM to detect Plasmodium falciparum, and HEX to detect P. ovale, $P$. vivax and P. malariae. Two P. falciparum samples and a mix of $P$. ovale, $P$. vivax and P. malariae were used as positive controls.

A nested PCR was performed for all the positive samples to validate the TaqMan assay. Two amplification reactions were carried out using cycling parameters of; $95^{\circ} \mathrm{C}$ for 5 min, 25 cycles of: $94^{\circ} \mathrm{C}$ for $30 \mathrm{sec}, 58^{\circ} \mathrm{C}$ for $2 \mathrm{~min}, 72^{\circ} \mathrm{C}$ for $2 \mathrm{~min}$, final extension at $72^{\circ} \mathrm{C}$ for $5 \mathrm{~min}$. Primers rPLU 5 , rPLU 6 were used during the first amplification reaction and P.fal1, P.fal2 were used for the second amplification reaction as described by [18].

\section{Insecticide susceptibility assays}

Following WHO procedures [19], $\mathrm{F}_{1}$ adults aged between 2-5 days were exposed for 1 hour to insecticide impregnated papers at WHO recommended concentrations. Eight insecticides belonging to the four major public health classes of insecticide were tested: the pyrethroids permethrin $(0.75 \%)$, deltamethrin $(0.05 \%)$, lambda-cyhalothrin $(0.05 \%)$ and $0.05 \%$ etofenprox; the organochlorines DDT (4\%) and dieldrin (4\%); the carbamate bendiocarb $(0.1 \%)$ and the organophosphate malathion (5\%). To further assess the extent of the susceptibility levels to all the insecticides, the $F_{1}$ population from Mityana was tested at two more exposure times of 30 and 20 minutes. Each test included control mosquitoes exposed to non treated papers.

\section{Transcription profiling of candidate resistance genes}

A quantitative Reverse Transcriptase PCR (qRT-PCR) was performed to assess the potential role of previously detected detoxification genes in An. funestus s.s. in the resistance observed in the collected An. funestus group mosquitoes. Total RNA was extracted from three batches (R1-R3) of $10 \mathrm{~F}_{1}$ females (2-5 days old) that survived after 30 minutes exposure to deltamethrin from Mityana. RNA was also extracted from 3 batches (C1-C3) of unexposed $F_{1}$ females that were used as control samples. Additionally, RNA was also extracted from 3 batches of 10 female mosquitoes from the fully susceptible An. funestus s.s strain FANG (S1-S3). RNA extraction, cDNA synthesis and qRT-PCR reactions were performed as previously reported [20,21]. Expression and fold change of each gene in resistant (R), control (C) and Fang susceptible (S) were calculated according to $2^{-\Delta \Delta \mathrm{CT}}$ method [22] following normalization with housekeeping genes RSP7 ribosomal protein S7 (AGAP010592) and the Actin 5C (AGAP000651) genes [20]. 


\section{Results}

\section{Mosquito collection and rearing}

A total of $1135 \mathrm{~F}_{0}$ mosquitoes were collected from the 3 villages combined with $97.97 \%$ belonging to the $A n$. funestus group. The highest number of $\mathrm{F}_{0} \mathrm{~s}$ came from Mityana $(n=688)$, whereas fewer samples were collected from Ntungamo $(n=196) .251 \quad F_{0} s$ were collected from Masindi. A total of $407 \mathrm{~F}_{1}$ egg batches were obtained for generating $\mathrm{F}_{1}$ adults in the insectary. $\mathrm{F}_{0} \mathrm{~s}$ reported here were identified as belonging to the An. funestus group after performing morphological assessment of collected samples. Other Anopheles species mainly from the $A n$. gambiae complex were also found in the study areas, notably; Masindi $\left(27 \mathrm{~F}_{0} \mathrm{~s}\right)$, Mityana $\left(12 \mathrm{~F}_{0} \mathrm{~s}\right)$ and Ntungamo $\left(19 \mathrm{~F}_{0} \mathrm{~s}\right)$. No attempts were made to obtain egg batches from non-members of the An. funestus group.

\section{Species Identification and distribution}

The species ID PCR performed indicated that An. funestus s.s. and $A n$. parensis, were the only members of the $A n$. funestus group collected from the three locations. An. parensis was the only species found in Mityana (Mt) and Ntungamo $(\mathrm{Nm})$, whereas for Masindi (Ms) both An. funestus and $A n$. parensis were present in a ratio of almost 1:1 (Table 1).

\section{Plasmodium infection rates}

A total of 140 samples (38 for An. funestus and 102 for An. parensis) from Mityana and Masindi were tested for sporozoite infection using TaqMan. In Mityana, all the 94 mosquitoes tested were $A n$. parensis exhibiting 4 Plasmodium falciparum infections (4.2\%), whereas in Masindi, all positive samples were $A n$. funestus s.s with an infection rate of $2 / 38$ (5.3\%), (Table 2). Plasmodium falciparum was the only detected malaria parasite species in all locations. However, when the nested PCR [18] was carried out, none of the An. parensis from Mityana was positive, whereas the two An. funestus s.s. from Masindi remained positive.

\section{Susceptibility to Insecticides}

A total of $1753 \mathrm{~F}_{1}$ adults from all the three sites combined were exposed to various insecticides. An. parensis from Mityana exhibited a full susceptibility to most insecticides

Table 1 Species composition by location after PCR species ID

\begin{tabular}{lccc}
\hline \multirow{2}{*}{ Description } & \multicolumn{3}{c}{ Location } \\
\cline { 2 - 4 } & Mityana & Ntungamo & Masindi \\
\hline Number of FOs Identified & 200 & 170 & 97 \\
Number of An. funestus & 0 & 0 & 42 \\
Number of An. parensis & 197 & 162 & 55 \\
\hline
\end{tabular}

3 negative PCR recorded in Mityana and 8 in Ntungamo. with $100 \%$ mortality observed for both males and females for the pyrethroids permethrin, lambda-cyhalothin and etofenprox. Similarly, a full susceptibility was observed for the organosphosphate malathion and the organochlorines dieldrin (Table 3; Figure 2). A reduced susceptibility was, however, observed for the pyrethroid deltamethrin (95 \pm $1.3 \%$ mortality for females), for DDT $(92 \pm 2.3 \%$ mortality for females) and also for the carbamate bendiocarb (96 \pm $0 \%$ mortality for females). A similar susceptibility profile was observed for the males (Table 3; Figure 2).

In Masindi where both An. parensis and An. funestus are present, resistance was observed to both type I (permethrin; $73 \pm 4.3 \%$ mortality for females) and II (deltamethrin; $53 \pm 2.5 \%$ mortality for females) pyrethroids and also to DDT $(69 \pm 2.3 \%$ mortality for females) (Table 3; Figure 2). Attempts were made to species identify survivors of insecticide exposure. Using PCR, all the 48 tested females were confirmed to be An. funestus s.s.. This indicates that the $A n$. parensis population in Masindi is similarly susceptible to these insecticides as the population from Mityana. Since there is a near ratio of $1: 1$ between the two species in Masindi, it can be deduced that the resistance level of An. funestus is probably twice the level recorded from these mortality rates (Table 3 ).

The $F_{1}$ adults raised from Ntungamo were not enough (only 56 females +65 males) for resistance profiling. However, mortality levels were not different from that observed in Mityana (Table 3).

After observation of high mortality ( $>92 \%$ for both males and females) in the Mityana population and for all the insecticides, two pools of 1200 Mityana $F_{1} s$ each, were exposed for 30 and 20 minutes respectively to further assess the extent of susceptibility in this population. There was no remarkable difference in mortality for the 30 min exposure with all the insecticides (Figure 3). The trend drastically changed after 20 minutes exposure, particularly with bendiocarb for which $>50 \%$ of the population survived after 24 hours. Mortality was remarkably lower with deltamethrin and DDT after 20 minutes exposure compared to $1 \mathrm{hr}$ and 30 minutes exposure (Figure 3).

\section{Trancription profiling of candidate resistance genes in An. parensis}

qRT-PCR analysis of the expression profiles in An. parensis of four candidate genes (CYP6M7, CYP9K1, CYP6P9 and CYP6AA4) previously associated with pyrethroid resistance in the sister species $A n$. funestus s.s. [20,23] was successfully carried out with primers originally designed for An. funestus. It is unknown whether the CYP6P9 is duplicated in $A n$. parensis as in An. funestus. Therefore, the primers used here for CYP6P9 were common to both duplicated CYP6P9a and CYP6P9b. Primers for all the four genes exhibited appropriate standard curve and amplification efficiency (between 90 and 110\%), suggesting 
Table 2 Malaria species distribution by location by TaqMan

\begin{tabular}{|c|c|c|c|c|c|}
\hline Location & & Number of F0s tested & Number of positives & $\%$ positivity & Detected Plasmodium species \\
\hline \multirow{2}{*}{ Masindi } & An. funestus & 38 & 2 & 4.3 & Plasmodium falciparum \\
\hline & An. parensis & 8 & none & 0.0 & - \\
\hline Mityana & An. parensis & 94 & 4 & 4.3 & Plasmodium falciparum \\
\hline
\end{tabular}

a high level of sequence conservation for these genes between the two species. None of the four genes was significantly over-expressed in the mosquitoes alive after $30 \mathrm{~min}$ exposure to deltamethrin (Resistant) than in those not exposed to insecticides (Control) (Figure 4). However, when the expression profiles of the four genes are compared with the fully susceptible FANG strain from An. funestus s.s., (Sus-FANG), the cytochrome P450 CYP9K1 is around 15 -fold over-expressed $(\mathrm{P}<0.001)$ in both resistant and control An. parensis samples than in FANG. The major gene associated with pyrethroid resistance in $A n$. funestus s.s., CYP6P9, is not significantly differentially expressed between the two species, while CYP6AA4 is rather significantly more expressed (7-fold; $\mathrm{P}<0.01)$ in the susceptible FANG strain than in An. parensis.

\section{Discussion}

Characterisation of members of the An. funestus group in Uganda has been very limited to date. In this study, we investigated the geographical distribution of members of this group, their contribution to malaria transmission and also their susceptibility to main public health insecticides.

\section{Species composition}

In this study, of the nine to eleven species of the $A n$. funestus group described to date, only An.parensis and An. funestus s.s were detected in Uganda. While the presence of $A n$. funestus s.s. is well documented [2,11] this is only the second report of $A n$. parensis. Indeed, An. parensis was previously identified in Western Uganda but only from outdoor collections [24]. Our findings show An. parensis to be abundantly present in Central (Mityana), South Western (Ntungamo) and North Western (Masindi) Uganda. An. funestus s.s is the main species of the group in the North and Eastern parts of Uganda from our collections and from previous studies carried out in these areas $[2,11]$. However, one cannot rule out the presence of other species of the group across the country because of the limited number of sites assessed. Additionally, the fact that this study only focused on indoor-resting

Table 3 Insecticide susceptibility levels by location after 1 hr exposure

\begin{tabular}{|c|c|c|c|c|c|c|c|}
\hline \multirow{2}{*}{ Insecticide } & \multirow{2}{*}{ Sex } & \multicolumn{2}{|c|}{ Mityana } & \multicolumn{2}{|c|}{ Masindi } & \multicolumn{2}{|c|}{ Ntungamo } \\
\hline & & $n$ & $\%$ mortality & $n$ & $\%$ mortality & $\bar{n}$ & \% mortality \\
\hline \multirow{2}{*}{ Permethrin } & $\mathrm{F}$ & 75 & $100 \pm 0$ & 93 & $73 \pm 4.3$ & 20 & $100 \pm 0$ \\
\hline & M & 75 & $100 \pm 0$ & 83 & $82 \pm 2.1$ & 22 & $100 \pm 0$ \\
\hline \multirow{2}{*}{ Deltamethrin } & $\mathrm{F}$ & 75 & $95 \pm 1.3$ & 75 & $53 \pm 2.5$ & 21 & $100 \pm 0$ \\
\hline & M & 75 & $97 \pm 1.3$ & 70 & $72 \pm 6.1$ & 26 & $100 \pm 0$ \\
\hline \multirow{2}{*}{ DDT } & $\mathrm{F}$ & 75 & $92 \pm 2.3$ & 90 & $69 \pm 2.3$ & 15 & $63 \pm 0$ \\
\hline & M & 75 & $93 \pm 1.3$ & 70 & $93 \pm 2.5$ & 17 & $100 \pm 0$ \\
\hline \multirow{2}{*}{ Lambda-cyhalothrin } & $\mathrm{F}$ & 75 & $100 \pm 0$ & / & - & / & - \\
\hline & M & 75 & $100 \pm 0$ & / & - & / & - \\
\hline \multirow{2}{*}{ Etofenprox } & $\mathrm{F}$ & 75 & $100 \pm 0$ & / & - & / & _- \\
\hline & M & 75 & $100 \pm 0$ & / & - & / & - \\
\hline \multirow{2}{*}{ Bendiocarb } & $\mathrm{F}$ & 75 & $96 \pm 0$ & / & - & / & - \\
\hline & M & 75 & $98.6 \pm 08$ & / & - & I & - \\
\hline \multirow{2}{*}{ Malathion } & $\mathrm{F}$ & 75 & $100 \pm 0$ & / & - & / & - \\
\hline & M & 75 & $100 \pm 0$ & / & - & / & - \\
\hline \multirow{2}{*}{ Dieldrin } & $\mathrm{F}$ & 75 & $100 \pm 0$ & I & - & I & - \\
\hline & M & 75 & $100 \pm 0$ & I & _ & / & _- \\
\hline \multirow{2}{*}{ Total } & $\mathrm{F}$ & 600 & & 258 & & 56 & \\
\hline & $M$ & 600 & & 223 & & 65 & \\
\hline
\end{tabular}

/: not tested. 


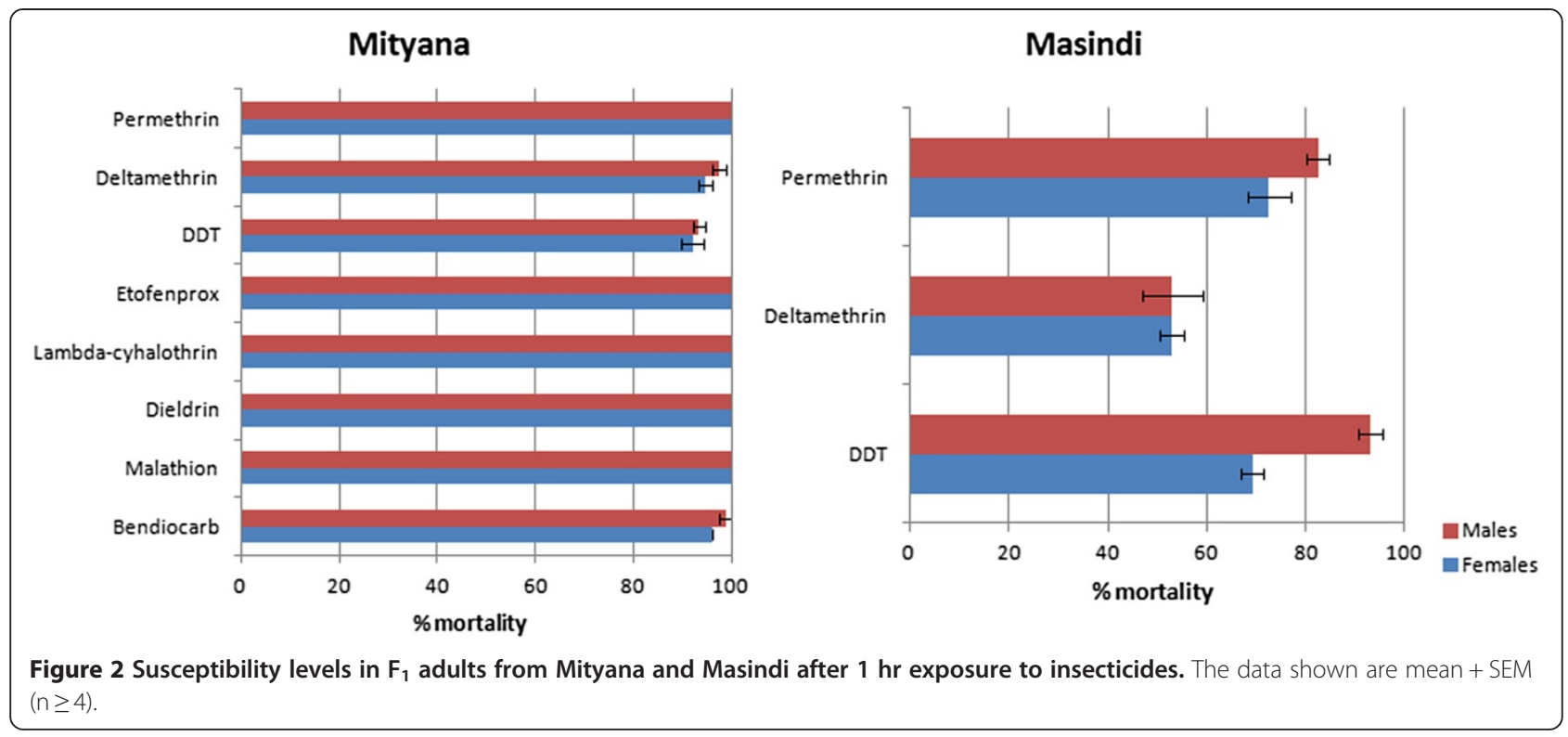

mosquitoes could have prevented the detection of exophilic species of the group.

In this study, An. parensis was found abundantly resting indoors similar to previous reports in other countries such as Kenya [25] and South Africa [26], indicating that this species which was previously described as mainly exophilic $[4,5,7]$, exhibits some plasticity in terms of its resting behaviour. The fact that An. funestus s.s and An. parensis, two species with such varying behaviour and vectorial capacity, as previously observed in Kenya [27], can be found indoors in sympatry is a serious concern to malaria vector control programs. An. parensis endophily as well as its sympatric occurrence with An. funestus s.s, such as in Masindi, is of significant interest since their varying behaviour [27] and infection rates could lead to misleading results on An. funestus s.s. if proper species identification is not carried out at the molecular level. This is the case in Masindi where the real level of resistance to insecticides in An. funestus has been underestimated because of the dilution from the sympatric susceptible An. parensis population. Additionally, even in areas where An. parensis is allopatric, its endophilic behaviour could lead control program managers to wrongly assume that An. funestus s.s. remains susceptible to insecticides. This may delay implementation of the much needed insecticide resistance management strategies to ensure the continued success of the insecticide-based control interventions. Mis-identification of vector species was already highlighted as one of the potential problems that led to vector control failure in the Garki project [28]. Our study further highlights how essential it is for every control program to ensure that molecular

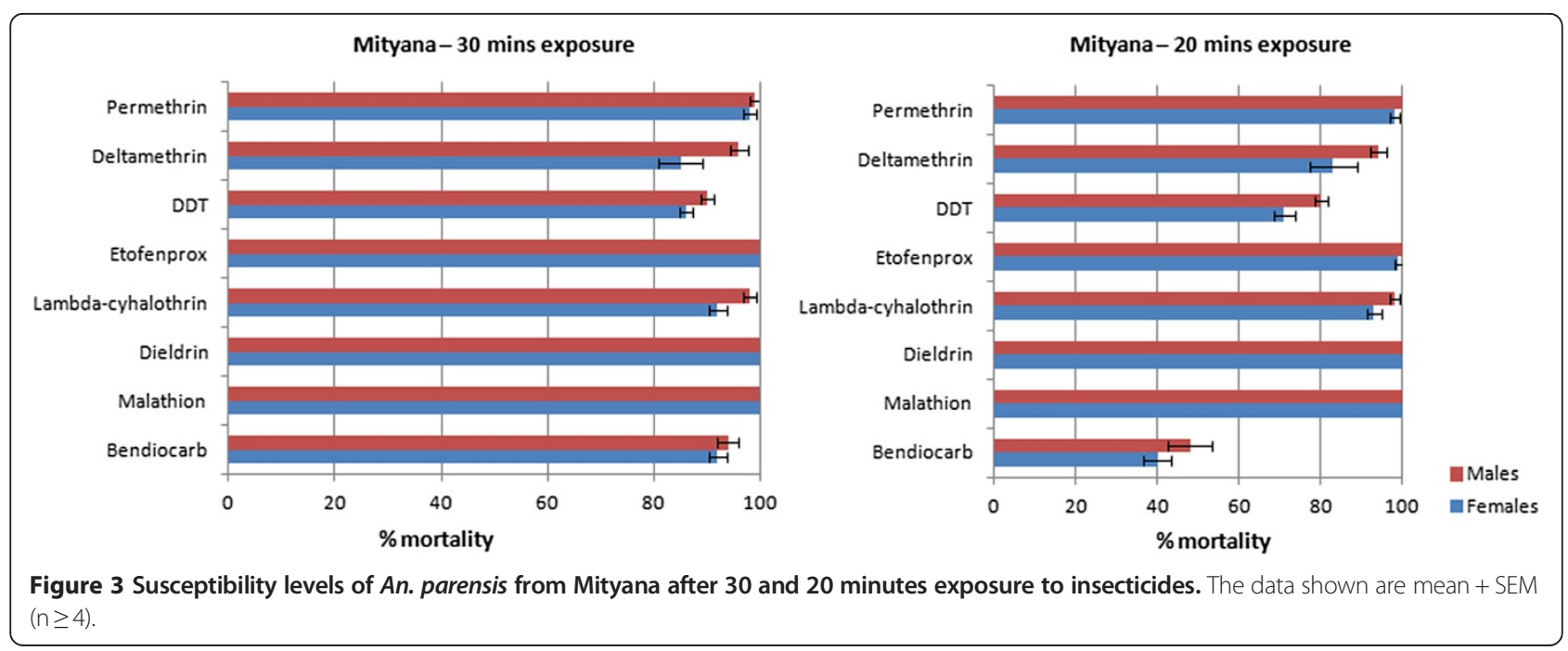




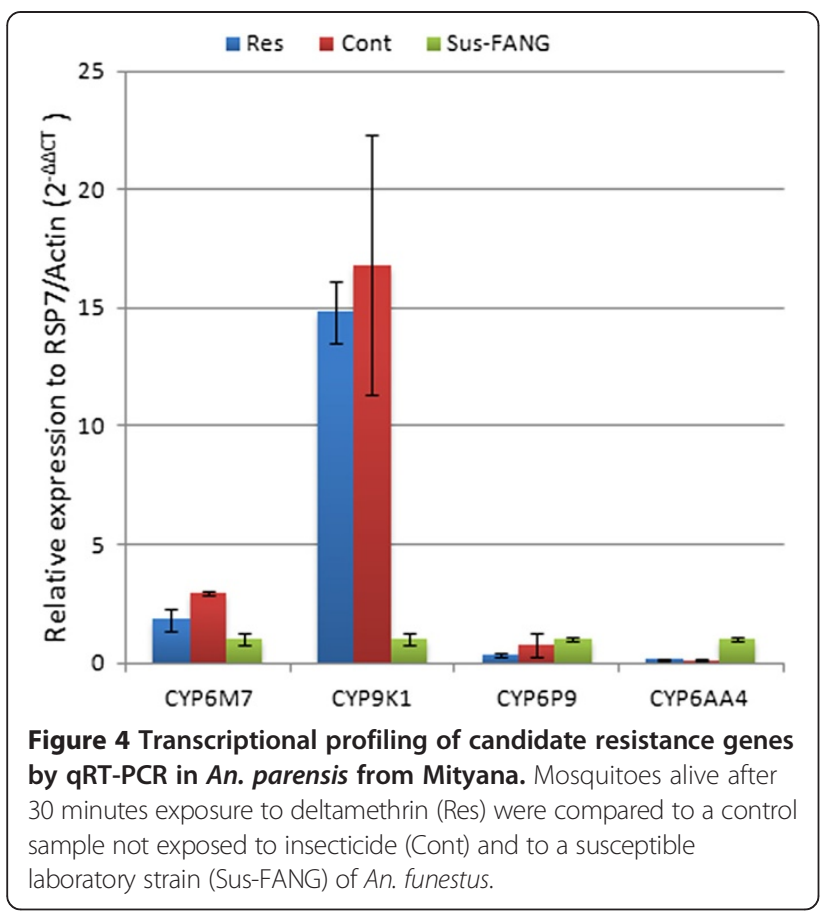

techniques together with morphological keys are used for reliable species identification during their surveillance activities.

\section{Sporozoite infection rate}

Previous reports $[25,29,30]$ have not incriminated $A n$. parensis in malaria transmission, however, given that a high number of indoor blood fed female An. parensis were collected, there was a need to establish whether it plays a role beside An. funestus in malaria transmission. This study confirms previous reports with the detection of sporozoite positive samples only in An. funestus s.s and not in An. parensis. The positive cases observed for An. parensis by TaqMan are probably due to false positives as also observed for this species in South Africa when using ELISA-CSP [26]. However, further investigations involving large scale screening of An. parensis for Plasmodia is highly recommended before this species is considered a non-malaria vector. The infection rate of $5.3 \%$ observed in An. funestus s.s. is similar to levels commonly reported for this species across Africa [13]. Although the source of blood meal was not tested in this study, the fact that cattle and other domestic animals were very few in Mityana, suggests that humans could have been the prime source of the blood meal for the collected females.

\section{Insecticide resistance}

In Mityana, the susceptibility of the An. parensis population to most insecticides is further supported by the low LT50 of this population for all insecticides, $(<20 \mathrm{~min}$, except for the carbamate bendiocarb). An. parensis susceptibility to insecticides in Uganda is similar to what was observed for this species in the KwaZulu Natal region of South Africa, where out of four tested insecticides, An. parensis was fully susceptible to three, specifically permethrin, DDT and bendiocarb [26]. However, a suspected resistance was observed against deltamethrin in the An. parensis population of KwaZulu Natal similar to the moderate resistance also observed for the same insecticide in Mityana. Overall, the similarity of susceptibility patterns between two populations of this species from East and Southern Africa suggests that $A n$. parensis is most likely still susceptible to most public health insecticides across its range of distribution in Africa. The susceptibility in An. parensis contrasts with the high resistance levels observed in $A n$. funestus in this study and other populations across Africa [14,31-34]. A similar contrast in insecticide resistance profiles was reported recently between An. gambiae s.s and An. arabiensis in Cameroon [35]. Such differences in susceptibility patterns suggest that An. parensis is only partially endophilic and under less selective pressure from public health control interventions than the predominantly endophilic An. funestus. Indeed, An. parensis has been found in the past to be mainly exophilic and exophagic [27] and therefore under less selection pressure than An. funestus. Since both species have similar breeding sites, it is likely that resistance may have been more selected in An. funestus due to its indoor resting behaviour, leading to exposure to public health insecticides, which may not have been the case for the more exophilic $A n$. parensis until recently. However, the endophilic behaviour displayed by An. parensis in this study is likely to increase the selection pressure on this species particularly with the recent scaling up of indoor residual spraying in Uganda. This situation is likely to lead to the rise of resistance in An. parensis in the future. Therefore, the susceptibility profile of this species should be monitored.

The low fold-change observed for four candidate resistance genes between the An. parensis samples that survived 30 min exposure to deltamethrin and the control nonexposed mosquitoes, further supports the overall susceptibility observed in this species contrary to An. funestus. The 15-fold over-expression of the cytochrome P450 gene, CYP9K1, in the An. parensis samples compared to the susceptible FANG strain could suggest that this gene may play a role in the observed deltamethrin resistance. However, because this difference could also be due by interspecies variations in gene expression, further work is needed to validate CYP9K1 involvement. It cannot be ruled out also that the genes responsible for the reduced susceptibility to deltamethrin in An. parensis could be different to that acting in An. funestus as observed between An. arabiensis and An. gambiae [36]. Indeed, it was recently shown that the metabolic resistance observed in a field population of $A n$. arabiensis in Chad is 
probably conferred by the CYP6P4 cytochrome $\mathrm{P} 450$ gene and not the CYP6P3 or CYP6M2 genes which are the major resistance genes in the sister species An. gambiae [36]. Therefore, other methods, notably genome-wide gene expression profiling, could be used to detect these An. parensis specific resistance genes.

\section{Conclusion}

The significant difference in the resistance profile and potential role of malaria transmission of An. parensis and An. funestus s.s reported in this study is a reminder of the importance of accurate species identification of malaria vectors in any vector control program. Such molecular identification should be part of any monitoring and surveillance activities in order to ensure that the impact of control interventions on vector populations is adequately assessed.

\section{Competing interests}

The authors declare that they have no competing interests.

\section{Authors' contributions}

CSW conceived and designed the research. CM and JB carried out the sample collection; CM and JMR performed the WHO bioassays; $\mathrm{CM}$ and $\mathrm{H}$ performed the molecular analyses; JB and LGM contributed toward data analysis and significant insights; CM and CSW drafted the manuscript with contribution from all the authors. All authors read and approved the final manuscript.

\section{Acknowledgement}

This work was supported by a Wellcome Trust Master Fellowship in Public Health and Tropical Medicine to CM (092794/Z/10/Z) and a Wellcome Trust Research Career Development Fellowship to CSW (083515/Z/07/Z).

\section{Author details}

${ }^{1}$ Vector Biology Department, Liverpool School of Tropical Medicine, Pembroke Place, Liverpool L3 5QA, UK. Uganda Virus Research Institute, Entebbe, Uganda.

Received: 25 October 2013 Accepted: 10 February 2014 Published: 17 February 2014

\section{References}

1. Yeka A, Gasasira A, Mpimbaza A, Achan J, Nankabirwa J, Nsobya S, Staedke SG, Donnelly MJ, Wabwire-Mangen F, Talisuna A, Dorsey G, Kamya MR Rosenthal PJ: Malaria in Uganda: challenges to control on the long road to elimination: I. Epidemiology and current control efforts. Acta Trop 2012, 121:184-195

2. Morgan JC, Irving H, Okedi LM, Steven A, Wondji CS: Pyrethroid resistance in an Anopheles funestus population from Uganda. PLoS One 2010, 5:e11872.

3. Cohuet A, Simard F, Toto JC, Kengne P, Coetzee M, Fontenille D: Species identification within the Anopheles funestus group of malaria vectors in Cameroon and evidence for a new species. Am J Trop Med Hyg 2003, 69:200-205.

4. Gillies MT, Coetzee M: A supplement to the Anophelinae of Africa south of the Sahara (Afrotropical region). Johannesburg: South African Institute for Medical Research; 1987

5. Gillies MT, De Meillon B: The anophelinae of Africa South of the Sahara. Johannesburg: The South African Institute for Medical Research; 1968.

6. Harbach RE: The classification of genus Anopheles (Diptera: Culicidae): a working hypothesis of phylogenetic relationships. Bull Entomol Res 2004, 94:537-553.

7. Spillings BL, Brooke BD, Koekemoer LL, Chiphwanya J, Coetzee M, Hunt RH: A new species concealed by Anopheles funestus Giles, a major malaria vector in Africa. Am J Trop Med Hyg 2009, 81:510-515.
8. Coetzee M, Koekemoer LL: Molecular systematics and insecticide resistance in the major African malaria vector Anopheles funestus. Annu Rev Entomol 2013, 58:393-412.

9. Dia I, Guelbeogo MW, Ayala D: Advances and Perspectives in the Study of the Malaria Mosquito Anopheles funestus. In Anopheles mosquitoes New insights into malaria vectors. Edited by Manguin S. In Tech Publisher; 2013:197-220

10. Wilkes TJ, Matola YG, Charlwood JD: Anopheles rivulorum, a vector of human malaria in Africa. Med Vet Entomol 1996, 10:108-110.

11. Okello PE, Van Bortel W, Byaruhanga AM, Correwyn A, Roelants P, Talisuna A, D'Alessandro U, Coosemans M: Variation in malaria transmission intensity in seven sites throughout Uganda. Am J Trop Med Hyg 2006, 75:219-225.

12. De Meillon B, Van Eeden G, Coetzee L, Coetzee M, Meiswinkel R, Du Toit C, Hansford C: Observations on a species of the Anopheles funestus subgroup, a suspected exophilic vector of malaria parasites in northeastern Transvaal, South Africa. Mosq News 1977, 37:657-661.

13. Coetzee M, Fontenille D: Advances in the study of Anopheles funestus, a major vector of malaria in Africa. Insect Biochem Mol Biol 2004, 34:599-605.

14. Cuamba N, Morgan JC, Irving H, Steven A, Wondji CS: High level of pyrethroid resistance in an Anopheles funestus population of the Chokwe District in Mozambique. PloS One 2010, 5:e11010.

15. Livak KJ: Organization and mapping of a sequence on the Drosophila melanogaster $\mathrm{X}$ and $\mathrm{Y}$ chromosomes that is transcribed during spermatogenesis. Genetics 1984, 107:611-634.

16. Koekemoer LL, Kamau L, Hunt RH, Coetzee M: A cocktail polymerase chain reaction assay to identify members of the Anopheles funestus (Diptera: Culicidae) group. Am J Trop Med Hyg 2002, 66:804-811.

17. Bass C, Nikou D, Blagborough AM, Vontas J, Sinden RE, Williamson MS, Field LM: PCR-based detection of Plasmodium in Anopheles mosquitoes: a comparison of a new high-throughput assay with existing methods. Malar J 2008, 7:177-177.

18. Snounou G, Viriyakosol S, Zhu XP, Jarra W, Pinheiro L, do Rosario VE, Thaithong S, Brown KN: High sensitivity of detection of human malaria parasites by the use of nested polymerase chain reaction. Mol Biochem Parasitol 1993, 61:315-320.

19. WHO: Test procedures for insecticide resistance montoring in malaria vectors, bio-efficacy and persistence of insecticides on treated surfaces. Geneva, Switzerland: World Health Organization; 1998.

20. Riveron JM, Irving H, Ndula M, Barnes KG, Ibrahim SS, Paine MJ, Wondji CS: Directionally selected cytochrome P450 alleles are driving the spread of pyrethroid resistance in the major malaria vector Anopheles funestus. Proc Natl Acad Sci U S A 2013, 110:252-257.

21. Jones CM, Machin C, Mohammed K, Majambere S, Ali AS, Khatib BO, McHa J, Ranson H, Kelly-Hope LA: Insecticide resistance in Culex quinquefasciatus from Zanzibar: implications for vector control programmes. Parasit Vectors 2012, 5:78.

22. Schmittgen TD, Livak KJ: Analyzing real-time PCR data by the comparative C(T) method. Nat Protoc 2008, 3:1101-1108.

23. Irving $H$, Riveron JM, Ibrahim SS, Lobo NF, Wondji CS: Positional cloning of rp2 QTL associates the P450 genes CYP6Z1, CYP6Z3 and CYP6M7 with pyrethroid resistance in the malaria vector Anopheles funestus. Heredity 2012, 109:383-392.

24. Echodu R, Okello-Onen J, Lutwama J, Enyaru J, Ocan R, Asaba R, Ajuga F, Rubaire-Akiiki C, Bradley D, Mutero C: Heterogeneity of anopheles mosquitoes in Nyabushozi County, Kiruhura district, Uganda. J Parasitol Vect Biol 2010, 2:28-34.

25. Kamau L, Koekemoer LL, Hunt RH, Coetzee M: Anopheles parensis: the main member of the Anopheles funestus species group found resting inside human dwellings in Mwea area of central Kenya toward the end of the rainy season. J Am Mosq Control Assoc 2003, 19:130-133.

26. Mouatcho JC, Hargreaves K, Koekemoer LL, Brooke BD, Oliver SV, Hunt RH, Coetzee M: Indoor collections of the Anopheles funestus group (Diptera: Culicidae) in sprayed houses in northern KwaZulu-Natal, South Africa. Malar J 2007, 6:30.

27. Gillies MT, Furlong M: An investigation into the behaviour of Anopheles parensis Gillies at Malindi on the Kenya coast. Bull Entomol Res 1964, 55:1-16.

28. Molineaux L, Gramiccia G: The Garki project: research on the epidemiology and control of malaria in the Sudan savanna of West Africa. Geneva; Albany, N.Y World Health Organization; WHO Publications Centre USA distributor; 1980. 
29. Awolola TS, Oyewole IO, Koekemoer LL, Coetzee M: Identification of three members of the Anopheles funestus (Diptera: Culicidae) group and their role in malaria transmission in two ecological zones in Nigeria. Trans $R$ Soc Trop Med Hyg 2005, 99:525-531.

30. Hargreaves K, Koekemoer LL, Brooke BD, Hunt RH, Mthembu J, Coetzee M: Anopheles funestus resistant to pyrethroid insecticides in South Africa. Med Vet Entomol 2000, 14:181-189.

31. Brooke BD, Kloke G, Hunt RH, Koekemoer LL, Temu EA, Taylor ME, Small G, Hemingway J, Coetzee M: Bioassay and biochemical analyses of insecticide resistance in southern African Anopheles funestus (Diptera: Culicidae). Bull Entomol Res 2001, 91:265-272.

32. Djouaka $R$, Irving $H$, Tukur Z, Wondji CS: Exploring mechanisms of multiple insecticide resistance in a population of the malaria vector anopheles funestus in Benin. PLoS One 2011, 6:e27760

33. Okoye PN, Brooke BD, Koekemoer LL, Hunt RH, Coetzee M: Characterisation of DDT, pyrethroid and carbamate resistance in Anopheles funestus from Obuasi, Ghana. Trans R Soc Trop Med Hyg 2008, 102:591-598.

34. Wondji CS, Coleman M, Kleinschmidt I, Mzilahowa T, Irving H, Ndula M, Rehman A, Morgan J, Barnes KG, Hemingway J: Impact of pyrethroid resistance on operational malaria control in Malawi. Proc Natl Acad SC U S A 2012, 109:19063-19070.

35. Nwane P, Etang J, Chouasmall Yi UM, Toto JC, Koffi A, Mimpfoundi R, Simard F: Multiple insecticide resistance mechanisms in Anopheles gambiae s.l. populations from Cameroon, Central Africa. Parasit Vectors 2013, 6:41.

36. Witzig C, Parry M, Morgan JC, Inving H, Steven A, Cuamba N, Kerah-Hinzoumbe C, Ranson H, Wondji CS: Genetic mapping identifies a major locus spanning P450 clusters associated with pyrethroid resistance in kdr-free Anopheles arabiensis from Chad. Heredity 2013, 110:389-397.

doi:10.1186/1756-3305-7-71

Cite this article as: Mulamba et al:: Contrasting Plasmodium infection rates and insecticide susceptibility profiles between the sympatric sibling species Anopheles parensis and Anopheles funestus s.s: a potential challenge for malaria vector control in Uganda. Parasites \& Vectors 2014 7:71.

\section{Submit your next manuscript to BioMed Central and take full advantage of:}

- Convenient online submission

- Thorough peer review

- No space constraints or color figure charges

- Immediate publication on acceptance

- Inclusion in PubMed, CAS, Scopus and Google Scholar

- Research which is freely available for redistribution 